\title{
Thématique et rêve d'un éternel globe-trotter.
}

Mélanges offerts à Shin-ichi Ichikawa. Textes recueillis et publiés par Shiro Fujii, Yoichi Sumi, Sakaé Tada. Tokyo : Comité Coordinateur des Mélanges Shin-ichi Ichikawa, achevé d'imprimer le 30 septembre 2003, par l'Imprimerie Kenkyûsha et distribué par la Librairie France Tosho, Tabata Building, 3 ème étage, 1-12-9, Nishi-Shinjuku, Shinjuku-ku, Tokyo 160-0023, Japon, tél. (81) 3-3346-0396, fax. (81) 3-3346-9154, 312 p.

\section{Henri Besse}

\section{(2) OpenEdition \\ Journals}

Édition électronique

URL : https://journals.openedition.org/dhfles/1286

DOI : $10.4000 /$ dhfles. 1286

ISSN : 2221-4038

Éditeur

Société Internationale pour l'Histoire du Français Langue Étrangère ou Seconde

Édition imprimée

Date de publication : 1 juin 2004

Pagination : 192-198

ISSN : 0992-7654

Référence électronique

Henri Besse, "Thématique et rêve d'un éternel globe-trotter. », Documents pour l'histoire du français langue étrangère ou seconde [En ligne], 32 | 2004, mis en ligne le 01 janvier 2012, consulté le 27 mai 2021.

URL : http://journals.openedition.org/dhfles/1286 ; DOI : https://doi.org/10.4000/dhfles.1286

Ce document a été généré automatiquement le 27 mai 2021.

(c) SIHFLES 


\section{Thématique et rêve d'un éternel globe- trotter.}

Mélanges offerts à Shin-ichi Ichikawa. Textes recueillis et publiés par Shiro Fujii, Yoichi Sumi, Sakaé Tada. Tokyo : Comité Coordinateur des Mélanges Shin-ichi Ichikawa, achevé d'imprimer le 30 septembre 2003, par l'Imprimerie Kenkyûsha et distribué par la Librairie France Tosho, Tabata Building, 3 ème étage, 1-12-9, Nishi-Shinjuku, Shinjuku-ku, Tokyo 160-0023, Japon, tél. (81) 3-3346-0396, fax. (81) 3-3346-9154, 312 p.

\section{Henri Besse}

1 Pourquoi faire un compte rendu, dans Documents, de ce beau volume à la reliure cartonnée à l'ancienne, aux titre et sous-titre en lettres d'or sur un fond toilé d'un vert profond, avec la photographie hors-texte du dédicataire protégée par un papier transparent comme on n'en trouve qu'au Japon, et dont les références éditoriales ne sont pas sans rappeler celles des ouvrages du XVIII siècle ? D'abord parce qu'on me l'a envoyé bien que, faute de temps, j'eusse décliné l'invitation à y contribuer, mais surtout parce qu'il me paraît à même d'intéresser les lecteurs de Documents, ne serait-ce qu'en leur permettant de faire connaissance avec des chercheurs de différents pays (de brèves biographies, en fin d'ouvrage, rappellent leurs parcours et leurs publications), dont les domaines de recherche sont plus ou moins familiers aux membres de la SIHFLES.

2 C'est que Shin-ichi Ichikawa s'est beaucoup investi, et pas que d'une manière réflexive, dans l'apprentissage de plusieurs langues européennes. Il a commencé par étudier le français à l'Université Waseda de Tokyo, avant d'y devenir professeur de littérature française et comparée, carrière qui l'a conduit à faire de nombreux séjours en France, en particulier à Montpellier où il a préparé son troisième cycle sous la direction de Jacques Proust. Mais il a fait aussi plusieurs séjours en Catalogne et en Castille, y apprenant l'espagnol. Il a également traduit en japonais des textes italiens et publié en anglais. Activité polyglotte et voyageuse qui l'a conduit à donner des conférences dans les pays francophones (France, Belgique, Canada, Suisse), mais aussi en Espagne, au Mexique, en Italie, aux États-Unis, en Hongrie et en Tchéquie. Ce qui suffirait à justifier 
le titre, assez peu académique, de l'ouvrage qui lui est dédié, n'était que les dix pages consacrées à sa "bibliographie personnelle » attestent que le "rêve " de "l'éternel globe-trotter " n'a pas été celui d'un dilettante à l'humeur vagabonde. Il a publié en japonais plusieurs ouvrages, dont un Jean-Jacques Rousseau et L'Univers des Encyclopédistes, ainsi qu'une cinquantaine d'articles, et il a effectué, seul ou en collaboration, une quinzaine de traductions en japonais d'ouvrages ou d'articles d'auteurs tant français qu'italiens. A quoi il faut ajouter une vingtaine d'articles en français, six en espagnol, un en italien et deux en anglais. Il s'est beaucoup intéressé aux Lumières, plus particulièrement aux représentions qu'elles se faisaient du Japon ou de la Chine (par ex., « Les mirages chinois et japonais chez Voltaire ») en les contrastant avec celles que les Japonais s'en faisaient (par ex., « Diderot au Japon à l'époque Meiji et Taîshô »). Mais aussi à l'introduction du français au Japon (par ex., « Le premier contact des Japonais avec la langue française et son destin avant la restauration de Meiji »), aux premières traductions de cette langue en japonais (par ex., «Du français au japonais par le truchement du hollandais. Difficultés rencontrées par nos premiers traducteurs : à propos de la Nouvelle Méthode des Langues Françoise et Hollandoise par Pieter Marin »), ou encore à la tradition de la traduction japonaise comparée à celle pratiquée en France (par ex., « Traduire Diderot en japonais » ou « Du nouveau sur la traduction au Japon comparée avec la tradition française »). Il s'est, en outre, intéressé aux romanciers japonais contemporains (par ex., à O. E. Kensaburô dans ses relations avec Jean-Paul Sartre). Et il a produit divers manuels destinés à l'enseignement du français au Japon : textes de Diderot ou de Mérimée annotés, la Correspondance privée en français, 194 Expressions idiomatiques, Pour bien rédiger en français. Shin-ichi Ichikawa est donc un esprit qui aime à sortir de l'ornière de sa spécialité académique, curieux de langues et de cultures autres que celles qui l'ont nativement façonné, et qui s'est voulu un médiateur ou un passeur entre le Japon et l'Europe occidentale. Les lecteurs de Documents qui souhaiteraient accéder à ses publications peuvent s'adresser directement à lui (sichikawa@waseda.jp).

3 L'ouvrage qui lui est dédié comprend vingt-cinq contributions (vingt-trois en français et deux en anglais, dont certaines traduites du japonais) qui toutes entretiennent des liens, parfois subtils mais réels, avec les préoccupations, ouvertes ou plus cachées, d'Ichikawa.

Deux d'entre elles portent sur ses propres travaux ou sur ce qu'on pourrait appeler son mode d'être. Une brève présentation par J. Renaud (professeur de khâgne au lycée Camille Guérin de Poitiers) de ses "articles en langues occidentales ", où il distingue "trois grands ensembles": les "études consacrées au XVIII" siècle français", celles portant sur la traduction et celles relatives à des écrivains japonais contemporains, études dont « le grand point » serait qu'elles éclairent ce qu'Ichikawa aime à qualifier de "distorsions », entre les " images du Japon en France [et les] images de la France au Japon ». Renaud y est aussi l'auteur d'un texte sur Francis Ponge, sans doute parce que ce dernier s'est voulu, à son propre dire, "combattant dans les rangs des lumières, comme on disait au grand siècle (le XVIII ${ }^{\mathrm{e}}$ )». La seconde contribution où Ichikawa est explicitement présent est qualifiée par son auteur, Fr. V. Tochon (University of Wisconsin, USA), de « réflexion hybride » où, passée une application un peu laborieuse $\mathrm{du}$ « carré sémiotique » à son thème, il traite de l'Émile de Jean-Jacques Rousseau et des rapports conflictuels que peuvent entretenir, dans une université américaine, l'enseignement de l'Éducation et l'enseignement des Lettres : «[...] un proverbe chinois (éducatif) énonce que lorsque le sage montre la lune, le sot regarde le doigt. Pour 
l'étudiant d'Éducation, il n'y a que la lune, pour l'étudiant de Lettres il n'y a que le doigt. " Tochon, natif de Genève et formé à la didactique au Canada, y narre ses démêlés à propos d'un plagiat commis par un étudiant qu'il a corrigé sans suivre les normes quasi juridiques ayant cours dans le Middle-West, assimilant non sans humour ses propres vicissitudes à celles de Jean-Jacques «trahi et honni de tous et de partout ", et regrettant de ne pas avoir l'aisance d'un Ichikawa naviguant d'une culture à une autre.

5 Sa spécialité initiale étant le XVIII siècle français, il n'est pas surprenant que plus de la moitié des contributions portent sur ce "grand siècle ». Trois traitent de D. Diderot et de l'Encyclopédie. Son premier maître en la matière, J. Proust (Université de Montpellier III) évoque ce qui a sans doute été la « première rencontre » de Diderot avec le Japon, l'Histoire de l'Église du Japon du jésuite Crasset qui lui fut offerte, alors qu'il avait quinze ans, comme second prix de composition en vers latins et en version latine. C. Roig (Universidad de Cantabria) analyse des traductions en espagnol, plus idéologiques que littéraires, du Supplément au voyage de Bougainville. Et Y. Sumi (Université Keio, Tokyo) s'interroge sur la place des "arts de mémoire » dans l'Encyclopédie. Cinq contributions traitent de J.-J. Rousseau. B. Didier (ENS Ulm) questionne le genre, entre journal intime et méditation philosophique, des Rêveries d'un promeneur solitaire. A. Mizubayashi (Université nationale des langues et civilisations étrangères de Tokyo) voit, dans la scène des Confessions où Rousseau décrit ce qui s'est passé dans un café de Fontainebleau alors qu'il y était venu à la répétition générale de son opéra Le Devin du village, une critique du discours social de cet «espace public », où Jean-Jacques ne voit pas «le premier des tribunaux» comme le voulait Voltaire, mais « un lieu de production de paroles mensongères ». S. Sato (Université Waseda) cerne le contexte intellectuel dans lequel Th. Hobbes et J.-J. Rousseau ont élaboré la théorie de l'État moderne. S. Tada (également de Waseda) revient sur « la notion d'âme dans l'Émile ». Et c'est aussi au traité d'éducation de Rousseau que Tochon (voir ci-dessus) consacre une partie de sa contribution.

6 A ces huit articles, qui constituent les deux premières parties de l'ouvrage, nous en ajouterons six autres qui se trouvent dans ses quatrième et cinquième parties. Celui de P. Casini (de La Sapienza, Rome), qui analyse comme un compromis entre Descartes et Newton, ou entre lois naturelles et lois positives, l'Esprit des lois selon Montesquieu. Celui de Y. Miyamoto (Université Hiroshima Jogakuinn), qui perçoit comme une contradiction le fait que Valmont et Merteuil réclament «à autrui ce qu'eux-mêmes ne pourront jamais accomplir ». Celui où $\mathrm{Cl}$. Reichler (Université de Lausanne) décrit un « jardin des lettres » près de Bâle comme « un théâtre des émotions » mettant en œuvre «la philosophie sensualiste proposée par Locke et développée par les philosophes des Lumières ». Celui où P. Zaborov (de la Maison Pouchkine de Saint-Pétersbourg) évoque ce que fut Louis-Philippe de Ségur. Ainsi qu'une analyse par J.-M. Goulemot (Université de Tours) des articles "Japon ", pris entre philosophie et sens commun, des principales encyclopédies françaises du XVIII ${ }^{e}$ siècle. Le rappel, par l'écrivain valdôtain L. Lexert, que ce siècle ne fut pas seulement celui de Voltaire, Diderot ou Rousseau, mais aussi celui d'un prince de Ligne, d'un chevalier de Nerciat, ou de nombre d'autres écrivains réputés du "second rayon». Ou encore, le texte de M. Voisin (Haute école de la communauté française du Hainaut) sur «Théophile Gautier et le XVIII siècle ». Le lien avec les travaux d'Ichikawa peut y sembler parfois ténu, il n'en est pas moins réel. Si, par exemple, Zaborov traite de ce Ségur (qui fut compagnon de Lafayette lors de son expédition américaine, ambassadeur de France auprès de Catherine II de Russie, grand- 
maître des cérémonies à la cour de Napoléon, polygraphe afin de nourrir sa famille au début de la Restauration avant d'y devenir pair de France), c'est sans doute parce qu'Ichikawa a écrit deux articles sur « Diderot et Kaditchev autour de Catherine II ».

7 Aux articles d'Ichikawa portant sur la traduction font écho la contribution de C. Roig (voir ci-dessus) et celle de F. Lafarga (Universitat de Barcelona) sur les traductions en espagnol de V. Hugo dans la seconde moitié du XIX siècle. Mais aussi celle de l'écrivain québécois J. Godbout, qui s'étonne des difficultés de son interprète «à expliquer à l'auditoire japonais le concept de frontière comme (il) l'entendai $(t)$ ». Et, d'une manière plus secrète, celles de H. Suematsu (Université du Kyûshu) sur les Immémoriaux de V. Segalen et de K. Tsunekawa (Université Hitotsbashi, Tokyo) sur Saint-John Perse, parce que l'une et l'autre s'interrogent sur l'art avec lequel on peut parvenir à rendre quasiment sensible en français les dialectes mélanésiens ou créoles qui sous-tendent et inspirent les textes de ces deux écrivains (ce qui me parait renvoyer à la traduction " suprême » selon Goethe, celle qui vaut « non à la place de l'autre mais en son lieu »). Restent trois contributions, qui ne traitent ni du XVIII ${ }^{\mathrm{e}}$ siècle ni de traduction, mais qui n'en sont pas moins liées aux préoccupations d'Ichikawa: celle de G. Campbell (Université de Calgary, Canada) qui rappelle la figure d'un militant des "Métis canadiens-français » au XIX ${ }^{e}$ siècle, écho sans doute aux deux articles qu'Ichikawa a publiés sur le français au Canada ; celle de P. Snowden (Université Waseda) portant sur l'introduction de l'anglais au Japon, qui forme une sorte de pendant à ses travaux sur l'introduction du français au Japon; et celle de S. Fujii (Université Ohu) qui traite de " la crise spirituelle et religieuse chez François Mauriac ", à la manière dont Ichikawa a pu s'intéresser à la littérature, japonaise et française, de son temps.

Ces contributions, dont la diversité rend hommage à la diversité des préoccupations de leur dédicataire, ne sont pas sans soulever d'autres hypothèses que celles qui y sont développées. En voici deux, parmi bien d'autres dont j'ai un peu rêvé - au sens que Rousseau prête à ce mot dans ses Rêveries - au cours de ma lecture. La première est liée au prosélytisme chrétien qui fut au Japon, au moins jusqu'au XVIII siècle, plus catholique que protestant. Snowden remarque que la première édition (1771) de l'Encyclopedia Britannica ne consacre qu'une seule ligne au Japon; Goulemot rappelle, à l'opposé, la place que lui accordent l'Encyclopédie et le dictionnaire de Moreri. Pourquoi cette différence de traitement? Sans doute parce que la propagande jésuite (Nicolas Poussin peint, en 1641 pour le Noviciat des jésuites de Paris, un « Saint François-Xavier rappelant à la vie la fille d'un habitant de Cangoxima au Japon ») avait popularisé dans les pays catholiques une image du Japon à laquelle échappait un pays non catholique comme le royaume britannique. Est-ce un hasard si ce sont des universitaires japonais qui traitent, dans ces "Mélanges offerts à Shin-ichi Ichikawa", de "l'âme " chez Rousseau, des "aventures du Libertin» (supposées immorales) chez Laclos ou de la "crise spirituelle " chez François Mauriac? L'introduction et le maintien des langues occidentales au Japon sont certes liés aux nécessités du commerce, mais aussi à ce prosélytisme chrétien que ces « Mélanges » n'exhibent guère. La seconde est liée à la traduction. On y apprend par exemple que les premiers traducteurs du Contrat social en japonais ont traduit le mot citoyen, tel que Rousseau l'emploie, par samouraï. On peut certes sourire de cette "distorsion ». Mais n'y a-t-il pas quelque chose d'exact dans cette équivalence entre un "citoyen", posé comme un idéal contestant la notion despotique de "sujet», et la figure archétypale du "samouraï» telle qu'elle a été construite par les oligarques de l'époque Meiji afin de restituer au Japon une identité échappant à l'hégémonisme occidental? Ne s'agit-il pas là d'une traduction de même 
type, militante ou idéologique, que celles du Supplément au voyage de Bougainville en Espagne aux XIX et $\mathrm{XX}^{\mathrm{e}}$ siècles ? Si Godbout avait su que le sens prêté actuellement au mot frontière (qu'on peut traverser à pied) ne date que de la fin du XVII ${ }^{\mathrm{e}}$ siècle, qu'il est lié entre autres à la politique expansionniste de Louis XIV, peut-être aurait-il été moins surpris par la perplexité de son interprète japonais ? Les « distorsions » des traducteurs sont parfois de véritables documents interculturels. Et pourquoi ne pas rêver d'un colloque qui se tiendrait au Japon sur le thème de l'introduction des principales langues de l'Europe de l'Ouest (portugais, espagnol, néerlandais, français, anglais, allemand) au Japon, et de l'histoire de leurs traductions successives dans la langue d'un pays trop bien protégé par la mer pour avoir eu besoin de la notion moderne (au sens que les historiens français continuent à donner à ce terme) de «frontière »?

\section{AUTEUR}

\section{HENRI BESSE}

ENS Lettres \& Sciences humaines - UMR CNRS 7597 